appropriateness of peri-operative plan and post-operative complications. No data was available on these outcomes prior to the advent of the foot MDT clinic. Results: Data from 12 clinics was analysed $(n=40)$. Patients had a median age of 66 years (IQR 27.5 years); $65 \%$ of patients were female and $35 \%$ of patients were male. The commonest rheumatological foot disease seen was rheumatoid arthritis $(67 \%)$, followed by psoriatic arthritis (15\%). All patients were treated with biologic or non-biologic DMARDs. Treatment outcomes were as follows: $27.5 \%$ were offered surgical treatment; $10 \%$ were offered intra-articular (IA) injections under ultrasound guidance; $10 \%$ were offered IA injections under general anaesthetic; $25 \%$ underwent specialist rheumatology podiatry, and the remaining $30 \%$ elected for a conservative approach after careful consideration of treatment options. Of those who were offered surgical treatment, $72 \%$ of patients were provided with a peri-operative plan which accorded with British Rheumatology Society (BSR) guidelines. Of those whom underwent surgery, one patient's surgical treatment was complicated by a post-operative infection; however, the peri-operative DMARD/biologic plan was not felt to be contributing factor.

Conclusion: The foot MDT clinic provides a comprehensive review of rheumatological foot conditions, with readily available access to a full range of treatment options. Co-location of all relevant professionals allows for real-time interdepartmental communication; shared decision making between clinicians and patients; avoids multiple appointments; reduces uncertainty with peri-operative planning as well as providing a cost-effective and efficacious service. Discrepancies in the peri-operative plan for medicines arose when the treating orthopaedic surgeon was not present in clinic. In these cases, the plan for surgical treatment was made outside of this clinic, without input from the treating rheumatologist. To improve concordance with BSR peri-operative medicine guidelines, it is recommended that all treatment decisions are made during the clinic, allowing input from all relevant partners. Informal feedback from patients commended the foot MDT, this shall be formalised through further qualitative data.

Disclosure of Interests: None declared

DOI: 10.1136/annrheumdis-2020-eular.343

\section{AB1346-HPR REAL-WORLD EFFECTIVENESS AND PERCEIVED USEFULNESS OF SYMPTOM CHECKERS IN RHEUMATOLOGY: INTERIM REPORT FROM THE PROSPECTIVE MULTICENTER BETTER STUDY}

J. Knitza ${ }^{1}$, J. Mohn¹, C. Bergmann ${ }^{1}$, E. Kampylafka ${ }^{1}$, M. Hagen ${ }^{1}$, D. Bohr ${ }^{1}$, E. Araujo ${ }^{1}$, M. Englbrecht ${ }^{2}$, D. Simon ${ }^{1}$, A. Kleyer ${ }^{1}$, T. Meinderink ${ }^{1}$, W. Vorbrüggen ${ }^{3}$, C. B. Von der Decken ${ }^{4,5}$, S. Kleinert ${ }^{6}$, A. Ramming ${ }^{1}$, J. Distler ${ }^{1}$ P. Bartz-Bazzanella ${ }^{5}$, G. Schett ${ }^{1}$, A. Hueber ${ }^{1,7}$, M. Welcker ${ }^{8}{ }^{1}$ Department of $^{-}$ Internal Medicine 3 - Rheumatology and Immunology, Universitätsklinikum Erlangen, Friedrich-Alexander-University Erlangen-Nürnberg (FAU), Erlangen Germany; ${ }^{2}$ Freelance Healthcare Data Scientist, Eckental, Germany; ${ }^{3}$ Verein zur Förderung der Rheumatologie e.V., Würselen, Germany; ${ }^{4} M V Z$ Stolberg, Stolberg, Germany; ${ }^{5}$ Klinik für Internistische Rheumatologie, Rhein-Maas Klinikum, Würselen, Germany; ${ }^{6}$ Rheumatologische Schwerpunktpraxis, Drs. Kleinert, Rapp, Ronneberger, Schuch u. Wendler, Rheumatology, Erlangen, Germany; ${ }^{7}$ Sektion Rheumatologie, Sozialstiftung Bamberg, Klinikum Bamberg, Bamberg, Germany; ${ }^{8} \mathrm{MVZ}$ für Rheumatologie Dr. Martin Welcker GmbH \& RheumaDatenRhePort (rhadar), Planegg, Germany

Background: Symptom checkers (SC) promise to reduce diagnostic delay, misdiagnosis and effectively guide patients through healthcare systems. They are increasingly used, however little evidence exists about their real-life effectiveness. Objectives: The aim of this study was to evaluate the diagnostic accuracy, usage time, usability and perceived usefulness of two promising SC, ADA (www. ada.com) and Rheport (www.rheport.de). Furthermore, symptom duration and previous symptom checking was recorded.

Methods: Cross-sectional interim clinical data from the first of three recruiting centers from the prospective, real-world, multicenter bETTeR-study (DKRS DRKS00017642) was used. Patients newly presenting to a secondary rheumatology outpatient clinic between September and December 2019 completed the ADA and Rheport SC. The time and answers were recorded and compared to the patient's actual diagnosis. ADA provides up to 5 disease suggestions, Rheport calculates a risk score for rheumatic musculoskeletal diseases (RMDs) ( $\geq 1=\mathrm{RMD}$ ). For both $\mathrm{SC}$ the sensitivity, specificity was calculated regarding RMDs. Furthermore, patients completed a survey evaluating the SC usability using the system usability scale (SUS), perceived usefulness, previous symptom checking and symptom duration.

Results: Of the 129 consecutive patients approached, 97 agreed to participate. $38 \%$ (37/97) of the presenting patients presented with an RMD (Figure 1). Mean symptom duration was 146 weeks and a mean number of 10 physician contacts occurred previously, to evaluate current symptoms. 56\% (54/96) had previously checked their symptoms on the internet using search engines, spending a mean of 6 hours. Rheport showed a sensitivity of $49 \%$ (18/37) and specificity of $58 \%$ (35/60) concerning RMDs. ADA's top 1 and top 5 disease suggestions concerning RMD showed a sensitivity of $43 \%(16 / 37)$ and $54 \%(20 / 37)$ and a specificity of $58 \%(35 / 60)$ and $52 \%(31 / 60)$, respectively. ADA listed the correct diagnosis of the patients with RMDs first or within the first 5 disease suggestions in $19 \%(7 / 37)$ and $30 \%(11 / 37)$, respectively. The average perceived usefulness fo checking symptoms using ADA, internet search engines and Rheport was 3.0 3.5 and 3.1 on a visual analog scale from $1-5$ (5=very useful). $61 \%(59 / 96)$ and $64 \%$ (61/96) would recommend using ADA and Rheport, respectively. The mean SUS score of ADA and Rheport was $72 / 100$ and 73/100. The mean usage time for ADA and Rheport was 8 and 9 minutes, respectively.

Conclusion: This is the first prospective, real-world, multicenter study evaluating the diagnostic accuracy and other features of two currently used SC in rheumatology. These interim results suggest that diagnostic accuracy is limited, however SC are well accepted among patients and in some cases, correct diagnosis can be provided out of the pocket within few minutes, saving valuable time.

Figure:

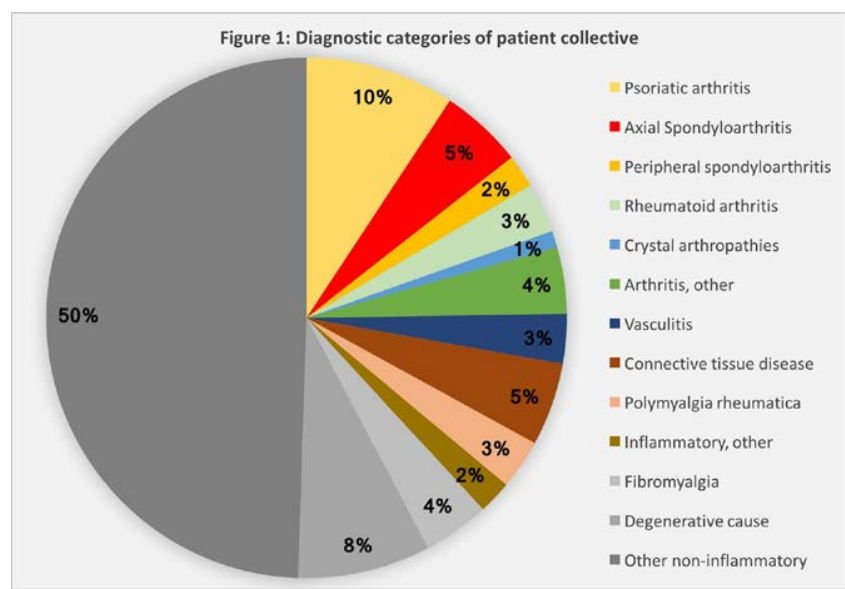

Acknowledgments: This study was supported by an unrestricted research grant from Novartis.

Disclosure of Interests: Johannes Knitza Grant/research support from: Research Grant: Novartis, Jacob Mohn: None declared, Christina Bergmann None declared, Eleni Kampylafka Speakers bureau: Novartis, BMS, Janssen, Melanie Hagen: None declared, Daniela Bohr: None declared, Elizabeth Araujo Speakers bureau: Novartis, Lilly, Abbott, Matthias Englbrecht Grant/research support from: Roche Pharma, Chugai Pharma Europe, Consultant of: AbbVie, Roche Pharma, RheumaDatenRhePort GbR, Speakers bureau: AbbVie, Celgene, Chugai Pharma Europe, Lilly, Mundipharma, Novartis, Pfizer, Roche Pharma, UCB, David Simon Grant/research support from: Else Kröner-Memorial Scholarship, Novartis, Consultant of: Novartis, Lilly, Arnd Kleyer Consultant of Lilly, Gilead, Novartis,Abbvie, Speakers bureau: Novartis, Lilly, Timo Meinderink: None declared, Wolfgang Vorbrüggen: None declared, Cay-Benedict von der Decken: None declared, Stefan Kleinert Shareholder of: Morphosys, Grant/ research support from: Novartis, Consultant of: Novartis, Speakers bureau: Abbvie, Novartis, Celgene, Roche, Chugai, Janssen, Andreas Ramming Grant/ research support from: Pfizer, Novartis, Consultant of: Boehringer Ingelheim Novartis, Gilead, Pfizer, Speakers bureau: Boehringer Ingelheim, Roche, Janssen, Jörg Distler Grant/research support from: Boehringer Ingelheim, Consultant of: Boehringer Ingelheim, Paid instructor for: Boehringer Ingelheim, Speakers bureau: Boehringer Ingelheim, Peter Bartz-Bazzanella: None declared, Georg Schett Speakers bureau: AbbVie, BMS, Celgene, Janssen, Eli Lilly, Novartis, Roche and UCB, Axel Hueber Grant/research support from: Novartis, Lilly, Pfizer, Consultant of: Abbvie, BMS, Celgene, Gilead, GSK, Lilly, Novartis, Speakers bureau: GSK, Lilly, Novartis, Martin Welcker Grant/research support from: Abbvie, Novartis, UCB, Hexal, BMS, Lilly, Roche, Celgene, Sanofi, Consultant of: Abbvie, Actelion, Aescu, Amgen, Celgene, Hexal, Janssen, Medac, Novartis Pfizer, Sanofi, UCB, Speakers bureau: Abbvie, Aescu, Amgen, Biogen, Berlin Chemie, Celgene, GSK, Hexal, Mylan, Novartis, Pfizer, UCB DOI: 10.1136/annrheumdis-2020-eular.1604

\section{AB1347-HPR DIGITAL SOLUTIONS TO AID SELF-MANAGEMENT: DEVELOPING A RHEUMATOLOGY APP FOR USE BY ANY PATIENT ATTENDING OUR DEPARTMENT}

K. Mackay ${ }^{1}$, H. Kelly ${ }^{2}$, M. Clemence ${ }^{1}$, R. Penford ${ }^{1} .{ }^{1}$ Torbay and South Devon NHS Foundation Trust, Torquay, United Kingdom; ${ }^{2}$ Torbay Hospital, Torquay, United Kingdom

Background: Managing complex rheumatological conditions requires information about the disease itself, treatments regimes and side effects. This is particularly important for those with a new diagnosis. 
A local patient focus group identified the need for trustworthy information, written and reviewed by their rheumatology team to aid self-management. They felt information should be readily accessible, in one place, to re-read / re-listen to, as required.

Objectives: To develop a Rheumatology App, for any patient attending our department, to support self-management.

Methods: We worked with a number of rheumatology patients, of differing ages, the Rheumatology multi-disciplinary team (MDT) and an industry partner, hci. digital, to develop our App, called 'Rheumatology Connect'.

We developed over 30 short videos, provided links to other valuable patient websites e.g. Versus Arthritis, NRAS, NASS, and wrote short articles, to provide basic headline information about various different diseases, common treatments e.g. Methotrexate. We provided easily accessible contact details and information about our clinic locations (including postcodes, bus links, car parking). Video content was written by the clinical authors and filmed by hci.digital.

Patient involvement in designing the layout was particularly important for the App to be easily navigable by all.

We launched the App, in June 2018, at a local patient educational conference. We encourage all patients on regular follow up to download it /use it and remind everyone about it in our clinic letters.

Results: Downloads have been ongoing since launch, with over 1953 downloads and 21930 page views, with peaks around the time of our monthly education sessions when we advertise the App to new patients.

Over $54 \%$ spent between 2 to $5+$ minutes in the App at a time. Most of the videos in the App are about 2 minutes long. Common hits include: videos on medications, various conditions, contact details, clinic locations, and services available. We have had a number of spontaneous contacts from patients saying how valuable they find the information we have given them.

The App aids efficiency by allowing direct phone calls to the correct member of the MDT e.g. rheumatology physio, rather than going through the secretaries. We are also encouraging direct email contact via the App. These aspects are helping us make our services more efficient.

Conclusion: The App continues to be downloaded and used by our patients. It has become an important part of our patient education efforts, especially for those with a new diagnosis.

We have had a significant increase in email contact from patients, allowing us to be more efficient by responding more quickly to patient queries, avoid additional telephone calls and avoid ansa-phone messages.

To date, the App continues to be downloaded by patients and we have received very positive feedback. Having surveyed the users, our next step is to develop interactive PROMS, a calendar reminder function and medicines management function as these have been identified as additions users would find helpful to manage their disease. We have raised funds to develop the App. The updated app should be available by May 2020

Download free from App store (search 'Rheumatology Connect') or go to www. rheumatologyconnect.info.

Disclosure of Interests: None declared

DOI: 10.1136/annrheumdis-2020-eular.5635

\section{AB1348-HPR TELEMEDICINE IN RHEUMATOLOGY: ACCEPTANCE BY PATIENTS AND MEDICAL PRACTITIONERS IN GERMANY (TELERHEUMABB) - INTERIM ANALYSIS}

F. Muehlensiepen $^{1,2,3}$, W. Marquardt ${ }^{2}$, J. Knitza $^{4}$, A. Hueber ${ }^{5}$, M. Welcker ${ }^{6,7}$. ${ }^{1}$ Medizinische Hochschule Brandenburg Theodor Fontane, Zentrum für Versorgungsforschung, Neuruppin, Germany; ${ }^{2} \mathrm{KV}$ Consult- und Managementgesellschaft $\mathrm{mbH}$, Potsdam, Germany; ${ }^{3}$ Witten/Herdecke University, Witten, Germany; ${ }^{4}$ Friedrich-Alexander University of ErlangenNürnberg, University Hospital Erlangen, Department of Internal Medicine 3 - Rheumatology and Immunology, Erlangen, Germany; ${ }^{5}$ Sozialstiftung Bamberg, Sektion Rheumatologie, Bamberg, Germany; ${ }^{6} \mathrm{MVZ}$ for RHEUMATOLOGY Dr. Martin Welcker GmbH, Planegg, Germany; ${ }^{7}$ RheumaDatenRhePort (rhadar), Planegg, Germany

Background: The number of rheumatologists in Germany does not cover the minimum medical need [1]. This implies long journeys and waiting periods for patients in the context of rheumatologists' consultations. International studies suggest that telemedicine could resolve these issues and support health care professionals in rheumatologic care $[2,3]$. So far, comprehensive sustainable approaches are still pending in Germany. By addressing the potential users, TeleRheumaBB investigates whether and how telemedicine can support rheumatologic care in Germany. The study is realized from Oct. 2017 until Jun. 2020. Objectives: The objectives of the study are to evaluate the acceptance and willingness of patients and medical practitioners (MP) to implement telemedicine into rheumatological care as well as to understand how telemedical approaches should be designed to be applied in daily practice.

Methods: The study design contains a mixed-methods approach, which combines qualitative and quantitative data. In the qualitative part of the study 28 in-depth interviews and 3 focus groups involving patients, rheumatologists, general practitioners and stakeholders were conducted. The quantitative part of the study comprised a questionnaire survey with rheumatologists $(n=48)$ and referrers $(n=444)$ and a second questionnaire survey with patients $(n=734)$.

Results:

Qualitative Data: The term "telemedicine" is filled with different meanings. Motives to implement telemedicine into daily practice are patient guidance, information, substantial time savings, desire of a modern practice etc. Reported risks of telemedicine are adverse impacts on the doctor-patient-relationship as well as low benefit with high effort.

Quantitative Data: $62 \%$ of the MPs' surveyed answered that they would like to use telemedical approaches. $81 \%$ of the participants supported doctor-doctor communication via telemedicine. Only $47 \%$ of the MPs' surveyed supported doctor-patients communication via telemedicine. MPs' state that there are barriers which prevent them from implementing telemedicine (89\%). These are among others acquisition of technology, administrative expenses and poor remuneration. $53 \%$ of the patients surveyed stated that they had heard the term telemedicine before. $30 \%$ of the participants answered that they would like to try out telemedicine. 38\% answered with "no" and 29\% with "do not know". Further results from the patient survey will be available in June 2020.

Conclusion: The study results show high acceptance rates of telemedicine regarding doctor-doctor communication. Doctor-patient communication via telemedicine is less accepted. However, MPs' are reporting obstacles preventing the implementation of telemedicine in rheumatology. In order to implement telemedicine in rheumatological care comprehensively, adequate conditions must be established in the German health care system.

\section{References:}

[1] Zink A, Braun J, Gromnica-Ihle E, Krause D, Lakomek HJ, Mau W, et al. Memorandum der Deutschen Gesellschaft für Rheumatologie zur Versorgungsqualität in der Rheumatologie - Update 2016. Zeitschrift für Rheumatologie. 2017;76(3):195-207.

[2] McDougall J, Ferruci E, Glover J, Fraenkel L (2017) Telerheumatologiy: A Systematic Review. Arthritis Care Res (Hoboken); 69(10):1546-1557.

[3] Piga M, Cangemi I, Mathieu A, Cauli A (2017) Telemedicine for patients with rheumatic diseases: Systematic review and proposal for research agenda Semin Arthritis Rheum 47(1):121-128.

Disclosure of Interests: Felix Muehlensiepen Grant/research support from: Novartis, Wenke Marquardt Grant/research support from: Novartis, Johannes Knitza Grant/research support from: Research Grant: Novartis, Axel Hueber Grant/research support from: Novartis, Lilly, Pfizer, EIT Health, EU-IMI, DFG, Universität Erlangen (EFI), Consultant of: Abbvie, BMS, Celgene, Gilead, GSK Lilly, Novartis, Speakers bureau: GSK, Lilly, Novartis, Martin Welcker Grant/ research support from: Abbvie, Novartis, UCB, Hexal, BMS, Lilly, Roche, Celgene, Sanofi, Consultant of: Abbvie, Actelion, Aescu, Amgen, Celgene, Hexal Janssen, Medac, Novartis, Pfizer, Sanofi, UCB, Speakers bureau: Abbvie, Aescu, Amgen, Biogen, Berlin Chemie, Celgene, GSK, Hexal, Mylan, Novartis, Pfizer UCB

DOI: 10.1136/annrheumdis-2020-eular.1865

\section{AB1349-HPR IMPLEMENTATION OF A GROUP EDUCATION SERVICE FOR RHEUMATOLOGY OUTPATIENTS COMMENCING BIOLOGIC THERAPY AT UNIVERSITY COLLEGE LONDON HOSPITAL (UCLH)}

M. Naja ${ }^{1}$, E. Hawkins ${ }^{1}$, S. Moore ${ }^{1}$, M. Castelino ${ }^{1} .{ }^{1}$ University College London Hospital, Rheumatology, London, United Kingdom

Background: Biologic therapies are commonly prescribed in a range of rheumatic conditions. From 2016 - 2018, a mean of 226 rheumatology outpatients at UCLH commenced biologic therapy per year for inflammatory arthritis alone ${ }^{1}$. Patient education is required prior to initiation. At UCLH this is delivered via individual appointments with specialist nurses, contributing to waiting times for nurse appointments and thus delays in starting treatment.

Objectives:

1.To evaluate patient satisfaction following group education.

2.To reduce the burden on specialist nurses delivering multiple individual sessions.

Methods: A once-weekly group education service was set up at UCLH. This comprised: an oral PowerPoint presentation; injection demonstration; question and answer session; and written leaflets. Patients were provided with pre- and post-session questionnaires to evaluate the service.

Results: Over the first five weeks of the service, 18 patients attended group edu cation (a median of 4 patients per session). Nine patients $(50 \%)$ had previously researched the medication via internet search engines. Patients' questions prior to the session were most commonly centred on side effects and long-term risks. All of the patients (100\%) felt that "most or all" of their questions were answered during the session. Patients gave their awareness of the reason for therapy a median score of $7 / 10$ pre-education; this rose to a median of $10 / 10$ post-education. The median score given for confidence to self-administer the drug was 